\title{
Does parental opinion differ from the health care team regarding cosmesis after hypospadias repair?

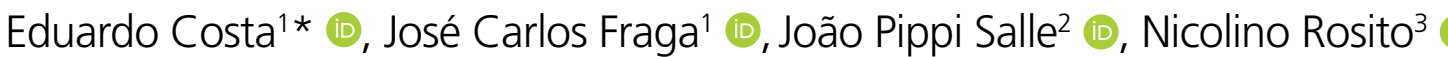

\begin{abstract}
SUMMARY
OBJECTIVE: Hypospadias is the most common malformation of the male genitalia. Surgical correction has traditionally focused on anatomic and functional outcomes, with less attention being paid to cosmetic results. Our purpose is to compare the cosmetic results of hypospadias repair among different groups of observers, namely the patient's family and the health care team, using photography and a simple rating scale.

METHODS: Prospective observational study included 9 boys undergoing Snodgrass hypospadias repair. Photographs of the penis taken before, immediately after, and six months after surgery were assessed by a panel of 15 observers (parents and health care team) and a scale including three questions with diagrams for comparison with the pictures was used. Observers also assigned an overall postoperative score for the cosmetic result.

RESULTS: Interobserver agreement was noted for the group of parents of other children with hypospadias regarding the shape of the glans $(k=0.404 ; p=0.008)$ and for the group of pediatric surgeons regarding the degree of residual curvature $(k=0.467 ; p=0.005)$. Two observers in the pediatrician group have indicated good performance in the assessment of residual curvature $(k=0.609 ; P=0.024)$. In the overall assessment of cosmetic outcomes, the highest scores were assigned by observers in the parents group and in the pediatrician group, while the pediatric surgeons group has one of the lowest scores $(p<0.001)$.

CONCLUSIONS: Photography appears to be suitable for documenting corrections of hypospadias regarding penile curvature, and postoperative cosmetic result. Surgeons seem more concerned about cosmesis than parents.

KEYWORDS: Hypospadias. Photography. Parents. Patient care team.
\end{abstract}

\section{INTRODUCTION}

Hypospadias is a common birth defect of the male genitalia, involving arrested development of the urethra, foreskin, and ventral surface of the penis ${ }^{1}$. In patients with hypospadias, the urethral meatus is located ventrally on the penis, with or without ventral penile curvature. The incidence of hypospadias is variable and depends on regional and ethnical differences. Some papers have shown increasing hypospadias rates and others have shown no increasing rates; but, currently, hypospadias is the most common malformation of the male genitaliaa ${ }^{1-5}$.

Different surgical techniques can be used to repair hypospadias. The main goals of surgery are the construction of a urethral meatus in orthotopic (glandular) position and correction of penile curvature, if present $t^{6}$. The correction of hypospadias has traditionally focused on anatomic and functional outcomes. However, a growing concern with body image has led

\footnotetext{
'Universidade Federal do Rio Grande do Sul; Hospital de Clínicas de Porto Alegre - Porto Alegre (RS), Brasil.

${ }^{2}$ Sidra Medical and Research Center - Doha, Qatar.

${ }^{3}$ Hospital de Clínicas de Porto Alegre - Porto Alegre (RS), Brasil.

*Corresponding author: eccosta@hcpa.edu.br

Conflicts of interest: the authors declare there are no conflicts of interest. Funding: none.

Received on August 10, 2020. Accepted on September 20, 2020.
} 
to greater attention being paid to cosmetic results. Assessment of cosmetic aspects is complicated by the absence of objective parameters. In addition, this assessment is often performed by reconstructive surgeons, who may not prioritize cosmetic results.

The use of photography could facilitate the assessment of the same patient by different people without exposing him. The objective of this study was to evaluate the cosmetic assessment of hypospadias repair by a panel of evaluators, including nurses, clinicians, surgeons, and parents, as well as to compare their opinion.

\section{METHODS}

This is a prospective observational study of consecutive children (6 months to 94 months of age/average age 14 months) undergoing hypospadias repair at a university hospital.

Photographs of the penis (frontal, lateral, and upper views) were taken before, immediately after surgery, and 6 months later. Photographs were taken using a Sony DSC-W30 digital camera, with 3x optical zoom and resolution of 6 megapixels. All photographs followed the same parameters: there was a focal distance of $20 \mathrm{~cm}$ and the lateral view pictures were always performed to the left side of the patient. A set of three pages containing three photographs each (preoperative, immediate postoperative, and 6 months postoperative photographs) was placed in a folder for each individual patient. The folder was then presented to a panel of 15 independent observers (not related to the patients and not involved in the study), divided into five groups: 3 pediatric surgeons $(\mathrm{S}), 3$ pediatricians $(\mathrm{P})$, 3 nurses $(\mathrm{N}), 3$ parents of other children with hypospadias $(\mathrm{PH})$, and 3 parents of children without hypospadias (PW). The observers were instructed to use a scale developed by our research group for evaluation of position of the meatus, shape of the glans, penile curvature, and general postoperative cosmetic result. The scale proposed has three objective questions; these questions were made based on an effort to harmonize parameters used to evaluate the images among observers. The findings were then compared to the impressions recorded by the investigator when examining the child after surgery. Physical examination performed by the investigator without access to the photographs was considered the gold standard.

The surgical technique described by Snodgrass et al. ${ }^{7}$ was used in all cases. All surgeries were performed as routine procedures by pediatric urology surgery professionals under the supervision of a staff surgeon.

Data analysis was performed using the Stata software, version $7.0^{8}$. Kappa coefficient was calculated for the comparison of individual observers. A generalized estimating equations (GEE) approach ${ }^{9}$ was used to verify whether there were differences in means among the five observer groups. After GEE was used, the Bonferroni test ${ }^{10}$ was used for multiple comparisons of means. The Bonferroni test was proposed by Fisher in order to analyze multiple comparisons. This consists in performing a t-test for each pair of means at an error rate by comparison. In this way, the Bonferroni test protects the error rate of the family of the tests. Significance level was set at $5 \%$. As the patients and observers were selected by convenience, and to avoid a very large folder, only nine patients were chosen.

\section{RESULTS}

Nine children treated consecutively for hypospadias were included in this study. At baseline, all patients had a hooded foreskin. Meatal position was subcoronal in five patients, distal penile in three, and midshaft penile in one. Three patients did not have penile curvature, five had mild/ moderate curvature (less than $45^{\circ}$ ) and only onehad severe curvature (greater than $45^{\circ}$ ). No patient received preoperative hormonal stimulation. Two patients developed postoperative subcoronal urethra cutaneous fistula and two had complete glans dehiscence. None presented residual penile curvature after surgery.

We obtained 144 evaluations using the hypospadias repair assessment scale. Table 1 describes interobserver agreement for the assessment of meatus position, penile shape, and penile curvature, based on the scale and photographic documentation. Moderate agreement $(\mathrm{k}=0.467 ; \mathrm{p}=0.005)$ was detected among $S$ observers regarding degree of residual curvature.

When contrasting the assessment made by each of the 15 individual observers against the gold standard (Table 2), we observed that, regarding the position of the urethral meatus, a good agreement rate was obtained for only one surgeon; nevertheless, this doesn't reach statistical significance. Regarding shape of the glans, agreement was slightly better; however, agreement was only significant for observer 1 in group $S \mathrm{p}<0.05)$. Despite a lower agreement rate, one PW observer showed a kappa of 0.357 (fair agreement) and $\mathrm{p}=0.015$. Evaluation of residual penile curvature revealed the highest levels of agreement in relation to the gold standard, with two observers (observers 2 and 3 in the $\mathrm{P}$ group) reaching $88.9 \%$. Both showed a kappa of 0.609 (moderate to substantial) and $\mathrm{p}=0.024$.

Regarding the overall rating of surgical results (question 4), the highest rates were attributed by the $\mathrm{PH}$ and $\mathrm{P}$ groups (9.52 and 8.67 respectively) (Figure 1). The $S$ group mean score was 7.98. The lowest mean score was given by the PW group (7.22). The Bonferroni test revealed statistical difference between the highest and lowest mean overall rates $(\mathrm{p}<0.001)$. 


\section{DISCUSSION}

Although photography has been increasingly used to evaluate hypospadias and the results of surgical correction, few studies have evaluated the usefulness of this tool in association with objective rating scales ${ }^{11-17}$. Baskin ${ }^{11}$ was the first to describe the results and complications of hypospadias surgery using photography, considering that photographic analysis could provide an unbiased assessment of cosmetic outcomes. El-Hout et al. ${ }^{12}$ also argued in favor of digital photography as a means for evaluation of hypospadias. Nevertheless, the results of that study showed poor inter-rater agreement in relation to urethral plate $(\mathrm{k}=0.06)$, highlighting the potential subjectivity of this evaluation, regardless of the level of surgeon experience.
Like us, Holland et al. ${ }^{13}$ have proposed a hypospadias objective scoring evaluation (HOSE), contemplating anatomical and functional aspects. However, HOSE is based on a total score resulting from the sum of all variables, with no individual analysis of each variable. The failure to stratify each variable independently may cause confusion if the results of one or more aspects are considered poor.

The present study, based on stratified results, revealed that observers had great difficulty in locating the correct position of the meatus on the photographs, when compared to the gold standard. The difficulty in determining the position of the urethral meatus on images may be related to the presence of postoperative edema as late as 6 months after surgery. Especially in patients who developed fistulas and wound or glans dehiscence,

Table 1. Kappa coefficient and p-value of agreement within each subgroup evaluating meatus position, shape of glans and penile curvature, using the proposed hypospadias repair assessment scale and photographic documentation.

\begin{tabular}{l|c|c|c|c|c|c|c|c} 
& Kappa & $p$ & & Kappa & $p$ & & Kappa & p \\
\hline Q1 & & & Q2 & & & Q3 & & \\
\hline observ_S & -0.044 & 0.584 & observ_S & -0.026 & 0.566 & observ_S & 0.467 & $0.005^{*}$ \\
\hline observ_P & -0.091 & 0.672 & observ_P & -0.344 & 0.973 & observ_P & 0.250 & 0.110 \\
\hline observ_N & -0.171 & 0.870 & observ_N & -0.179 & 0.871 & observ_N & 0.161 & 0.188 \\
\hline observ_PH & 0.030 & 0.431 & observ_PH & 0.404 & $0.008^{*}$ & observ_PH & 0.071 & 0.349 \\
\hline observ_PW & -0.115 & 0.781 & observ_PW & -0.103 & 0.756 & observ_PW & -0.267 & 0.956
\end{tabular}

Q1: question 1 (meatus position); Q2: question 2 (shape of glans); Q3: question 3 (penile curvature); Observ_S: pediatric surgeons; observ_P: pediatricians; observ_N: nurses; observ_PH: parents of other children with hypospadias; observ_PW: parents of children without hypospadias; Kappa Agreement: <0 less than chance agreement, $0.01-0.20$ slight agreement, $0.21-0.40$ fair agreement, $0.41-0.60$ moderate agreement, $0.61-0.80$ substantial agreement and 0.81-0.99 almost perfect agreement; *Indicates statistical significance.

Table 2. Agreement between observers using the proposed hypospadias repair assessment scale and photographic documentation versus clinical assessment by surgeons performing the surgery (gold standard).

\begin{tabular}{|c|c|c|c|c|c|c|c|}
\hline $\begin{array}{l}\text { Question } \\
\text { and } \\
\text { observer }\end{array}$ & $\begin{array}{c}\text { Agreement } \\
(\%)\end{array}$ & Kappa & $p$ & $\begin{array}{l}\text { Question } \\
\text { and } \\
\text { observer }\end{array}$ & $\begin{array}{c}\text { Agreement } \\
(\%)\end{array}$ & Kappa & $p$ \\
\hline q1gs q1s1 & 66.7 & 0.270 & 0.118 & q2gs q2ph2 & 66.7 & 0.342 & 0.147 \\
\hline q2gs q2s1 & 66.7 & 0.426 & $0.037^{*}$ & q2gs q2ph3 & 55.6 & 0.265 & 0.134 \\
\hline q2gs q2p1 & 66.7 & 0.386 & 0.083 & q2gs q2pw3 & 55.6 & 0.357 & $0.015^{*}$ \\
\hline q2gs q2p3 & 55.6 & 0.182 & 0.171 & q3gs q3s3 & 77.8 & 0.400 & 0.067 \\
\hline q2gs q2n1 & 66.7 & 0.372 & 0.076 & q3gs q3p2 & 88.9 & 0.609 & 0.024 * \\
\hline q2gs q2n3 & 66.7 & 0.342 & 0.147 & q3gs q3p3 & 88.9 & 0.609 & 0.024 * \\
\hline q2gs q2ph1 & 55.6 & 0.217 & 0.208 & q3gs q3n1 & 77.8 & -0.125 & 0.646 \\
\hline
\end{tabular}

q1: question 1 (meatus position); q2: question 2 (shape of glans); q3: question 3 (penile curvature); gs: gold standard; s1: observer_pediatric surgeon 1; s3: observer_pediatric surgeon 3; p1: observer_pediatrician 1; p2: observer_pediatrician 2; p3: observer_pediatrician 3; n1: observer_nurse 1; n3: observer_nurse 3; ph1: observer_parent of other child with hypospadias 1; ph2: observer_parent of other child with hypospadias 2; ph3: observer_parent of other child with hypospadias 3; pw3: observer parent of child without hypospadias; *Indicates statistical significance. 
the complication site was the meatal location. Another misleading factor that is not always clear on images is wound or glans dehiscence. These complications were observed in two of our patients.

Also, regarding aesthetic appraisal derived from anatomical aspects, Ververidis et al. ${ }^{14}$ have proposed an objective assessment of cosmetic results based on slide images projected onto a lecture-theater screen for approximately 1 hour, considering meatus, glans, shaft, and overall appearance. The authors compared two different methods used for surgical correction: Snodgrass technique and Mathieu/Duckett repair. The Snodgrass et al. ${ }^{7}$ technique (which was used in our patients) was considered the best approach. Despite using photography and evaluating cosmetic results, this study cannot be compared to ours, because the authors set different goals: the results for two different techniques were compared and a totally subjective score was used. In our study, the three first questions corresponded to anatomical analysis, facilitating the uniformity of response. Another difficulty posed by the referred study concerns the projection of images. In our study, the observers could evaluate the images individually for as long as deemed necessary.

In the study by Weber et al. ${ }^{15}$, the Pediatric Penile Perception Score (PPPS), proposed by the authors after hypospadias repair, was applied to patients, parents of patients, a control group of patients, a control group of parents, and urologists. Like the study by Ververidis et al. ${ }^{14}$, a subjective evaluation was performed by asking about the degree of satisfaction with the outcome of surgical repair. The authors concluded that the PPPS was an appropriate instrument to assess penile self-perception in children after hypospadias repair. Our study also assessed anatomical variables and, even though we did not include patient self-assessment, we evaluated the opinion of

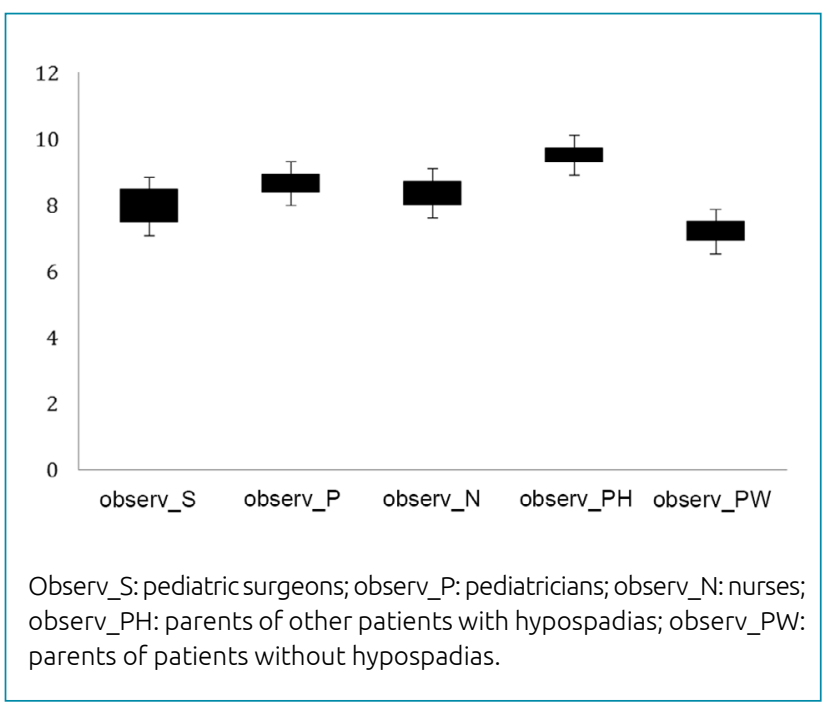

Figure 1. Mean and confidence interval for scores in question 4, according to observer group. parents of other children with hypospadias. This group of parents ended up assigning the highest mean scores for final penile appearance. Surgery outcome was also highly evaluated by pediatricians, demonstrating that these professionals, who are the primary referring medical practitioners, were satisfied with the final results.

Regarding only the opinions of surgeons, Van der Toorn et al. ${ }^{16}$ proposed the Hypospadias Objective Penile Evaluation (HOPE) score, which is an objective scoring system for evaluation of cosmetic appearance in hypospadias correction. The HOPE scoring system evaluates six correctable items: the position of meatus, shape of meatus, shape of glans, shape of penile skin, and penile axis, including penile torsion and penile curvature. The study reported strong intra-rater reproducibility. Furthermore, the HOPE score may be useful to compare cosmetic outcomes obtained with different techniques. If we consider only our $S$ group, there was high agreement regarding the degree of residual curvature and meatus position.

Haid et al. ${ }^{17}$ compared parents' and surgeons' opinions using PPPS and HOPE scores. Parents had worse results than surgeons regarding cosmesis using those scores. But there was no difference between groups when considering the general appearance of the penis. In 1996, Mureau et al. ${ }^{18}$ brought to attention that patients weren't satisfied with their penile appearance after hypospadias repair, particularly when compared to the surgeon's view. A systematic review published in $2016^{19}$ illustrated what probably is the most important measure to assure a good result: keeping patients in a long follow-up, as Springer ${ }^{6}$ has already mentioned. Both papers showed that results sometimes are questioned. Snodgrass et al. ${ }^{20}$ indicated that parents' and surgeons' opinions of cosmetic were similar, when comparing hypospadias repair and elective circumcision. Our study suggested parents were more satisfied with cosmetic results than surgeons. It is important to remember that those parents didn't analyze their sons' results, so they didn't have any personal issues involved like previous papers cited before.

Scarpa et al. ${ }^{21}$ have compared objective vs. subjective assessment of cosmetic results after distal hypospadias repair. There was an excellent level of agreement among four observers (objective observer, operating surgeon, independent surgeon and parent of child). Like us, those authors also detected the highest scores among the parents of patients with hypospadias.

Another important finding from our study is that two pediatricians achieved very good agreement with the gold standard regarding residual curvature, a better performance than that of surgeons individually. This finding suggests that the professionals who are most likely to refer patients to surgeons are well trained with respect to detection of penile curvature, at least in our institution. 
A possible limitation of the present work is the small number of patients. However, the number of evaluations - $144-$ is more satisfactory. Each patient had nine pictures that were analyzed 15 times (panel of observers) and also by the investigator, and this total number was sufficient to ensure statistical relevance. Although we observed an unusually high number of complications after hypospadias repairs, these were not important because all observers had the same picture for analysis, and the focus of this study was on the agreement among different observers.

Another limitation of this paper is that our scale wasn't validated and it was prone to being subjective, since, because we had parents analyzing pictures, we tried to make it as simple it could be. In the other hand, it looks very easy to apply, considering it has only four questions.

\section{CONCLUSIONS}

This study shows that photography may be a good means of documenting corrections of hypospadias regarding the shape of the glans, penile curvature, and postoperative cosmetic outcomes. However, photography was not adequate to assess the shape of the glans and the position of the meatus, especially in the presence of small fistulas. Regarding this aspect, upper view photographs are not necessary, because they did not adequately show meatus position and simply meant three additional pictures per patient. Among all observers, parents of other children with hypospadias expressed the highest satisfaction with the cosmetic outcome of hypospadias repair. This finding could suggest that surgeons are more concerned with aesthetic aspects than patients' parents. At least, this trend is perceived according to data obtained at our institution, and although we used a small sample, it was analyzed in a meticulous way. This conclusion is important to help make surgeons less concerned with aesthetic issues. Additionally, this paper suggested that pediatricians were well informed to identify penile malformations, mainly hypospadias associated with penile curvature, thus leading to appropriate referral for surgical repair. More data is necessary to assure those findings.

\section{ACKNOWLEDGEMENTS}

This paper was conceptualized and written at the Pediatric Surgery Service of the Hospital de Clínicas de Porto Alegre (HCPA) in Porto Alegre, Brasil. It's part of a master's thesis pertaining to the Postgraduate Program in Surgery at Universidade Federal do Rio Grande do Sul (UFRGS).

Ethical approval: All procedures performed in studies involving human participants were in accordance with the ethical standards of the institutional and/or national research committee and with the 1964 Helsinki declaration and its later amendments or comparable ethical standards.

Our IRB approval is 07649 .

Informed consent: informed consent was obtained from all individual participants included in the study.

\section{AUTHORS" CONTRIBUTIONS}

EC: Conceptualization, Data Curation, Formal Analysis, Project Administration, Writing - Original Draft, Writing Review \& Editing. JPS: Conceptualization, Writing - Original Draft, Writing - Review \& Editing. JCF: Conceptualization, Formal Analysis, Writing - Original Draft, Writing - Review \& Editing. NR: Data Curation.

\section{REFERENCES}

1. Bergman JE, Loane M, Vrijheid M, Pierini A, Nijman RJ, Addor $M C$, et al. Epidemiology of hypospadias in Europe: a registrybased study. World J Urol. 2015;33(12):2159-67. https://doi. org/10.1007/s00345-015-1507-6

2. Leung AK, Robson WL. Hypospadias: an update. Asian J Androl. 2007;9(1):16-22. https://doi.org/10.1111/j.17457262.2007.00243.x

3. Baskin LS, Ebbers MB. Hypospadias: anatomy, etiology, and technique. J Pediatr Surg. 2006;41(3):463-72. https://doi. org/10.1016/j.jpedsurg.2005.11.059

4. Mouriquand PD, Mure PY. Current concepts in hypospadiology. BJU Int. 2004;93(Suppl)3:26-34. https://doi.org/10.1111/j.1464410X.2004.04706.x
5. Springer A, van den Heijkant M, Baumann S. Worldwide prevalence of hypospadias. J Pediatr Urol. 2016;12(3):152. e1-7. https://doi.org/ 10.1016/j.jpurol.2015.12.002

6. Springer A. Assessment of outcome in hypospadias surgery: a review. Front Pediatr. 2014;2:2. https://doi.org/10.3389/ fped.2014.00002

7. Snodgrass W, Koyle M, Manzoni G, Hurwitz R, Caldamone A, Ehrlich R. Tubularized incised plate hypospadias repair: results of a multicenter experience. J Urol. 1996;156(2 Pt 2):839-41. https://doi.org/10.1097/00005392-199608001-00082

8. Renfro CG [editor]. A compendium of existing econometric software packages. J Econ Soc Meas. 2004;29:359-409. https://doi.org/10.3233/JEM-2004-0225 
9. Liang KY, Zeger SL. Longitudinal data analysis using generalized linear models. Biometrika. 1986;73(1):13-22. https://doi. org/10.1093/biomet/73.1.13

10. Dunn OJ. Multiple comparisons among means. J Am Stat Assoc. 1961;56(293):52-64. https://doi.org/10.2307/2282330

11. Baskin L. Hypospadias: a critical analysis of cosmetic outcomes using photography. BJU Int. 2001;87(6):534-9. https://doi. org/10.1046/j.1464-410x.2001.00092.x

12. El-Hout Y, Braga LH, Pippi Salle JL, Moore K, Bägli DJ, Lorenzo AJ. Assessment of urethral plate appearance through digital photography: do pediatric urologists agree in their visual impressions of the urethral plate in children with hypospadias? J Pediatr Urol. 2010;6(3):294-300. https://doi.org/10.1016/j. jpurol.2009.09.003.

13. Holland AJ, Smith GH, Ross Fl, Cass DT. HOSE: an objective scoring system for evaluating the results of hypospadias surgery. BJU Int. 2001;88(3):255-8. https://doi.org/10.1046/j.1464410x.2001.02280.x

14. Ververidis M, Dickson AP, Gough DC. An objective assessment of the results of hypospadias surgery. BJU Int. 2005;96(1):135-9. https://doi.org/10.1111/j.1464-410X.2005.05582.x

15. Weber DM, Schönbucher VB, Landolt MA, Gobet R. The Pediatric Penile Perception Score: an instrument for patient self-assessment and surgeon evaluation after hypospadias repair. J Urol. 2008;180(3):1080-4. https://doi.org/10.1016/j. juro.2008.05.060

16. van der Toorn F, de Jong TP, de Gier RP, Callewaert PR, van der Horst EH, Steffens MG, et al. Introducing the HOPE
(Hypospadias Objective Penile Evaluation)-score: a validation study of an objective scoring system for evaluating cosmetic appearance in hypospadias patients. J Pediatr Urol. 2013;9(6 Pt B):1006-16. https://doi.org/10.1016/j.jpurol.2013.01.015

17. Haid B, Becker T, Koen M, Berger C, Strasser C, Roesch J, et al. Penile appearance after hypospadias correction from a parent's point of view: comparison of the hypospadias objective penile evaluation score and parents penile perception score. J Ped Urol. 2016;12(1):33.e1-7. https://doi.org/10.1016/j. jpurol.2015.10.010

18. Mureau MA, Slijper FM, Slob AK, Verhulst FC, Nijman RJ. Satisfaction with penile appearance after hypospadia surgery: the patient and surgeon view. J Urol. 1996;155(2):703-6. PMID: 8558709

19. Long CJ, Canning DA. Hypospadias: are we as good as we think when we correct proximal hypospadias? J Ped Urol. 2016;12(4):196.e1-5. https://doi.org/10.1016/j. jpurol.2016.05.002

20. Snodgrass W, Ziada A, Yucel S, Gupta A. Comparison of outcomes of tubularized incised plate hypospadias repair and circumcision: a questionnaire-based survey of parents and surgeon. J Pediatr Urol. 2008;4(4):250-4. https://doi. org/10.1016/j.jpurol.2007.12.007

21. Scarpa M, Castagnetti M, Musi L, Rigamonti W. Is objective assessment of cosmetic results after distal hypospadias repair superior to subjective assessment? J Pediatr Urol. 2009;5(2):110-3. https://doi.org/10.1016/j. jpurol.2008.10.004 